\title{
Comparative genomic analysis of a multiple antimicrobial resistant enterotoxigenic $E$. coli 0157 lineage from Australian pigs
}

Ethan Wyrsch ${ }^{1 \dagger}$, Piklu Roy Chowdhury ${ }^{1,2+}$, Sam Abraham ${ }^{2,3}$, Jerran Santos ${ }^{1}$, Aaron E Darling ${ }^{1}$, lan G Charles ${ }^{1}$, Toni A Chapman ${ }^{2+}$ and Steven P Djordjevic ${ }^{1^{*+}}$

\begin{abstract}
Background: Enterotoxigenic Escherichia coli (ETEC) are a major economic threat to pig production globally, with serogroups O8, O9, O45, O101, O138, O139, O141, 0149 and 0157 implicated as the leading diarrhoeal pathogens affecting pigs below four weeks of age. A multiple antimicrobial resistant ETEC 0157 (O157 SvETEC) representative of $\mathrm{O} 157$ isolates from a pig farm in New South Wales, Australia that experienced repeated bouts of pre- and post-weaning diarrhoea resulting in multiple fatalities was characterized here. Enterohaemorrhagic E. coli (EHEC) O157:H7 cause both sporadic and widespread outbreaks of foodborne disease, predominantly have a ruminant origin and belong to the ST11 clonal complex. Here, for the first time, we conducted comparative genomic analyses of two epidemiologically-unrelated porcine, disease-causing ETEC O157; E. coli O157 SVETEC and E. coli O157:K88 734/3, and examined their phylogenetic relationship with EHEC O157:H7.
\end{abstract}

Results: 0157 SVETEC and O157:K88 734/3 belong to a novel sequence type (ST4245) that comprises part of the ST23 complex and are genetically distinct from EHEC 0157. Comparative phylogenetic analysis using PhyloSift shows that E. coli O157 SvETEC and E. coli O157:K88 734/3 group into a single clade and are most similar to the extraintestinal avian pathogenic Escherichia coli (APEC) isolate O78 that clusters within the ST23 complex. Genome content was highly similar between E. coli O157 SvETEC, O157:K88 734/3 and APEC O78, with variability predominantly limited to laterally acquired elements, including prophages, plasmids and antimicrobial resistance gene loci. Putative ETEC virulence factors, including the toxins STb and LT and the K88 (F4) adhesin, were conserved between O157 SVETEC and O157:K88 734/3. The O157 SVETEC isolate also encoded the heat stable enterotoxin STa and a second allele of STb, whilst a prophage within O157:K88 734/3 encoded the serum survival gene bor. Both isolates harbor a large repertoire of antibiotic resistance genes but their association with mobile elements remains undetermined.

Conclusions: We present an analysis of the first draft genome sequences of two epidemiologically-unrelated, pathogenic ETEC O157. E. coli O157 SvETEC and E. coli O157:K88 734/3 belong to the ST23 complex and are phylogenetically distinct to EHEC O157 lineages that reside within the ST11 complex.

Keywords: Enterotoxigenic Escherichia coli, Diarrhoeal disease, Swine, Porcine, 0157

\footnotetext{
* Correspondence: Steven.Djordjevic@uts.edu.au

'Equal contributors

${ }^{1}$ The ithree institute, University of Technology Sydney, P.O. Box 123,

Broadway, NSW 2007, Australia

Full list of author information is available at the end of the article
}

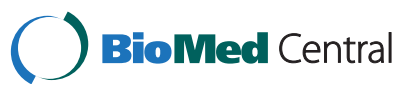

(C) 2015 Wyrsch et al.; licensee BioMed Central. This is an Open Access article distributed under the terms of the Creative Commons Attribution License (http://creativecommons.org/licenses/by/4.0), which permits unrestricted use, distribution, and reproduction in any medium, provided the original work is properly credited. The Creative Commons Public Domain Dedication waiver (http://creativecommons.org/publicdomain/zero/1.0/) applies to the data made available in this article unless otherwise stated. 


\section{Background}

Enterotoxigenic Escherichia coli (ETEC) are a leading cause of neonatal, pre-weaning and post-weaning diarrhoea (PWD) in pigs. Significant economic losses are incurred as a result of mortalities, reduced growth rates in survivors and medication costs. Antimicrobials have been used to treat infections but the emergence of multiple drug resistant variants poses a serious challenge in controlling ETEC infections in swine production. Dietary zinc supplements, phage therapy, probiotics, antibody dietary formulations and breeding programs to generate swine stock expressing gut epithelial receptors that are not recognised by ETEC fimbriae all represent alternate strategies used by swine producers to control ETEC [1], however these pathogens continue to pose a constant threat to farms.

ETEC serogroups O8, O9, O45, O101, O138, O139, O141, O149 and O157 are implicated in diarrhoeal disease during pig development [2-4]. Porcine enterotoxigenic E. coli $\mathrm{O} 157$ are associated with disease of the small intestine and mediate neonatal, pre- and postweaning diarrhoea in piglets. This is in contrast to the mode of pathogenesis displayed by EHEC O157, which is associated with food-borne human illness causing diarrhoea and enterohaemorrhagic disease of the large intestine [5-7]. Multiple evolutionary lineages of E. coli O157 [8] with different lineages encoding variants of the $\mathrm{H}$-antigen genes, and two distinct $r f b E$ alleles within the O157-antigen biosynthesis gene cluster, have been described. In addition, reports of serogroup O157 isolates as ETEC [9] and EPEC [10,11] is indicative of a wider evolutionary range of pathogenic E. coli O157, sourced from multiple hosts including humans, pigs and cattle.

Features separating porcine ETEC from other diarrhoeagenic $E$. coli include the presence of the pig-specific fimbrial adhesins F4 (K88), F5, F6, F18 and F41 that facilitate adherence to porcine gut epithelium and toxins including the heat-stable enterotoxins STa and STb, and a heat-labile enterotoxin (LT) that induce diarrhoea [1,12]. Molecular epidemiological studies indicate that other putative virulence genes are also expressed by ETEC causing ND and PWD, including EAST1 toxin, haemolysins, autotransporters, outer membrane proteins, siderophores and additional iron acquisition factors [2,13,14]. Many putative virulence genes are present only in subsets of clinical ETEC isolates suggesting that different virulence gene combinations may contribute to the range of clinical symptoms observed in ETEC infections. Additionally, ETEC readily acquire virulence and antimicrobial resistance genes by lateral gene transfer and strain variants containing new combinations of virulence and antimicrobial resistance genes are continually evolving globally [13,15-18].

In 2008 a piggery in Australia sustained major economic losses from an outbreak of pre- and post-weaning diarrhoea caused by enterotoxigenic Escherichia coli (ETEC) with an O157 serogroup. A representative of the ETEC outbreak is isolate E. coli O157 SvETEC. E. coli O157 SvETEC caused pre- and post-weaning diarrhoea in pigs between 7-15 days of age, affecting farrowing as well as weaner sheds. Severely affected pigs died suddenly with little or no diarrhoea. Gilts were shown to shed the pathogen but remained free of symptoms during the outbreak. Various antimicrobial and management strategies were implemented and all failed to control the ETEC infection in the pig herd. The outbreak resulted in a significant loss of piglets, over a long duration of time and over multiple sheds. Eventually all stock were removed, pens cleaned and restocked. We present here a comparative genomic analyses of O157 SvETEC with a historical ETEC O157 isolate from Australia (O157:K88 734/3) sampled in the 1990s from a clinical neonatal diarrhoea specimen $[4,19]$.

In this study, a combination of bioinformatics and genome sequencing methodologies was used to perform a comparative and phylogenetic analysis of strains $\mathrm{O} 157$ SvETEC and O157:K88 734/3. These are the first ETEC O157 genomes deposited in public databases. Our analyses provide insight into the evolution of ETEC O157 isolates in Australian swine populations revealing that they are phylogenetically distinct to other $E$. coli isolates of serogroup O157. We identified a suite of putative virulence factors, antimicrobial resistance genes and mobile genetic elements in the two porcine $\mathrm{O} 157$ isolates. In addition, we highlight novel variability between these two related pathogens.

\section{Methods}

Strains, isolation, culture conditions and serogrouping Isolate O157 SvETEC (previously ETEC 95 [19]) was isolated in 2008 from the faeces of an affected piglet from a commercial farm in New South Wales (Australia) that experienced repeated bouts of pre- and post-weaning diarrhoea and high mortality. Isolate O157:K88 734/3 (previously ETEC 24 [19]) was sampled in the 1990s from a clinical submission of common neonatal diarrhoea. Isolates were initially characterised at the NSW Department of Primary Industries Elizabeth Macarthur Agricultural Institute (EMAI) in Menangle $[4,13,19]$. The strains were sent to the ithree institute at the University of Technology Sydney (UTS), as stab cultures and thereafter regularly cultured in LB broth supplemented with ampicillin $\left(50 \mu \mathrm{g} \mathrm{ml}^{-1}\right)$ with shaking at $200 \mathrm{rpm}$ at $37^{\circ} \mathrm{C}$ for approximately 16 hours.

\section{Antimicrobial resistance phenotyping}

At EMAI, strains were screened against 18 antimicrobial agents by disc diffusion using the calibrated dichotomous susceptibility (CDS) test Australia, as reported previously [4]. The following antimicrobials were tested: ampicillin $(25 \mu \mathrm{g})$, amoxicillin/clavulanate $(60 \mu \mathrm{g})$, ticarcillin/ 
clavulanic acid $(85 \mu \mathrm{g})$, cefalexin $(100 \mu \mathrm{g})$, cefoxitin $(30 \mu \mathrm{g})$, cefotaxime $(5 \mu \mathrm{g})$, cefepime $(10 \mu \mathrm{g})$, nalidixic acid $(30 \mu \mathrm{g})$, ciprofloxacin $(2.5 \mu \mathrm{g})$, imipenem $(10 \mu \mathrm{g})$, sulphafurazole $(300 \mu \mathrm{g})$, trimethoprim $(5 \mu \mathrm{g})$, tetracycline $(10 \mu \mathrm{g})$, apramycin $(15 \mu \mathrm{g})$, neomycin $(30 \mu \mathrm{g})$, gentamicin $(10 \mu \mathrm{g})$, azithromycin $(15 \mu \mathrm{g})$ and chloramphenicol $(30 \mu \mathrm{g})$.

\section{Genomic DNA extraction}

Sequencing quality gDNA was extracted from $2 \mathrm{~mL}$ of each overnight culture using a DNeasy Blood and Tissue Kit (Qiagen) following manufacturer's recommendations.

\section{Whole genome sequencing, assembly, annotation and phylogenetic analysis}

Sequencing was performed at the UTS in-house Next Generation Sequencing facility using a bench top Illumina MiSeq ${ }^{\circ}$ sequencer and $\mathrm{MiSeq}^{\circ} \mathrm{V} 3$ chemistry. Sequencing libraries were prepared with $0.5 \mathrm{ng}$ of gDNA following the manufacturer's protocol for the Nextera ${ }^{\circ}$ XT library preparation kit (Illumina). Sequencing with the MiSeq ${ }^{\circ}$ sequencer generated 250 nucleotide (nt) long paired end reads of the libraries representing each sample. The quality of the sequence reads was assessed using a locally downloaded version (0.10.1) of FastQC (http://www.bioinformatics.babraham.ac.uk/projects/fastqc/) software and assembled using the A5-miseq de novo assembly pipeline [20] revised to process reads up to $500 \mathrm{nt}$ long [21]. Scaffolds over $1000 \mathrm{nt}$ in length were included in the whole genome sequence analysis. Whole Genome Shotgun sequences were deposited at DDBJ/EMBL/ GenBank under the accession numbers JPPP00000000 for E. coli O157 SvETEC and JPQX00000000 for E. coli O157:K88 734/3. A preliminary annotation of each genome was generated using the automated annotation software RAST [22] and the annotation of antimicrobial resistance genes was performed using the Resistance Gene Identifier (RGI) Version 2 on the Comprehensive Antibiotic Resistance Database website [23]. Individual genes of interest, including those annotated by RAST and the RGI, were manually interrogated using NCBI's BLASTn and BLASTp tools. Insertion sequences (IS) and open reading frames (ORFs) were identified using the online tools IS Finder (https://www-is.biotoul.fr//) and ORF Finder (http://www.ncbi.nlm.nih.gov/gorf/gorf.html) respectively. Phage associated gene clusters within the genomic scaffolds were initially identified using the PHAST [24] server. Genomic scaffolds with positive PHAST hits were further verified to be phage-associated in the RAST annotation output and using BLASTn and BLASTp analysis.

An alignment of phylogenetic marker genes was constructed using PhyloSift [25] and a tree was then inferred using FastTree2 [26]. The publicly available FastTree2 software is unable to resolve branches in the phylogeny shorter than $1 \times 10^{-5}$ substitutions per site. Our dataset appeared to have several short branches; therefore we modified the FastTree2 software to improve short branch resolution and applied it to our dataset. The output was visualised in FigTree v1.4.2 (http://tree.bio.ed.ac.uk/ software/figtree/). The O157 SvETEC and O157:K88 $734 / 3$ genomes were analysed alongside 40 complete $E$. coli genomes, 3 complete Shigella spp. genomes, 33 draft E. coli $\mathrm{O} 157$ genomes and 2 draft E. coli APEC O78 genomes from the NCBI GenBank database. The Klebsiella pneumonia 342 and Salmonella enterica subsp. enterica serovar Heidelberg str. 41578 genome sequences were included in this analysis as out-groups to confirm the validity of the method; however these sequences were removed from the final phylogenetic tree to facilitate visualizing the fine-scale relationships among E. coli.

\section{Comparative genomic and MLST analysis}

Comparative genomics used tools available in MAUVE version 2.3.1 [27]. The MAUVE Move Contigs tool was used to tile scaffolds generated by the de novo A5-miseq assembler against the reference E. coli APEC O78 finished genome. From this, the best alignment was chosen based on the highest weight score, an indicator of whether the predicted rearrangement exists, and lowest number of Locally Collinear Blocks (LCBs). Scaffolds that tiled against the finished APEC O78 genome were sorted and identified in this study as the subset representing the 'core' genome. The subset of scaffolds that did not align against the finished APEC O78 genome were designated the 'accessory' genome. The progressiveMauve module was used for comparative analysis of the genomes and to generate the figure. Regions of interest identified from whole genome comparisons were further analysed using iterative BLASTn and BLASTp searches.

The PubMLST (http://pubmlst.org/) database was used to identify the sequence type of the isolates using the Achtman E. coli MLST scheme [28] (http://mlst.warwick. ac.uk/mlst/).

\section{Results}

\section{Whole genome sequence statistics and phylogenetics}

De-novo assembly of the O157 SvETEC genome generated 236 scaffolds with 60 -fold coverage and a predicted genome size of 5547789 nt. The N50 value for the assembly was 96352 nt. For O157:K88 734/3, de-novo assembly generated 226 scaffolds with 78 -fold coverage, a predicted genome size of $5449663 \mathrm{nt}$ and an N50 value of $91578 \mathrm{nt}$. In both cases, $50 \%$ of the respective genomes were assembled into the largest 18 scaffolds. Scaffolds were sorted into cohorts putatively representing the core and accessory genomes of O157 SvETEC and O157:K88 734/3 using the criteria described above. The O157 SvETEC core genome consisted of 96 scaffolds totalling 4789945 nt while the O157:K88 734/3 core genome comprised 94 scaffolds 
totalling $4809185 \mathrm{nt}$. The accessory genome of O157 SvETEC was $738983 \mathrm{nt}$ in length and was represented in 112 scaffolds while the O157:K88 734/3 accessory sequence totalled $616231 \mathrm{nt}$ in 97 scaffolds.

Phylogenetic analysis using the assembled genome sequences was performed to gain insight into the evolution of ETEC O157 isolates in Australian swine populations. In-silico identification of the genes used in the updated Clermont et al. phylotyping method [29] determined that O157 SvETEC and O157:K88 734/3 belonged to phylogroup C. This concurred with previously reported data [4].

The PhyloSift phylogenetic analysis was performed to examine the swine isolates in the context of $E$. coli population structure. Figure 1 shows that neither E. coli $\mathrm{O} 157$ SvETEC nor E. coli O157:K88 734/3 clustered with the other E. coli serogroup O157 isolates and grouped together into a single clade with the most closely related completely closed reference genome being an avian pathogenic $E$. coli (APEC) of serogroup O78 [GenBank: CP004009]. Notably, the subclade included a variety of other isolates including S. sonnei 53G [GenBank:HE616528], known pathogenic E. coli serogroups such as O111 [GenBank:AP010960], O104 [GenBank:CP003301] and O26 [GenBank:AP010953] and a draft E. coli O157:H43 genome sequence [GenBank:AHZD02000001]. E. coli O157 draft and complete genomes as well as E. coli $\mathrm{O} 55$ genomes grouped together as a large clade in the tree, clustering with a confidence value of 1 . The findings of our phylogenetic analysis were supported by eMLST analysis in which the O157 SvETEC and O157:K88 734/3 isolates represented a novel sequence type, ST4245 [4]. For comparative purposes, we also sequence typed APEC O78, the closest neighbour of the porcine $\mathrm{O} 157$ isolates and found it to be ST23, a member of the same clonal complex.

\section{Comparative genomics}

The scaffolds representing the core O157 SvETEC genome had 4,741 predicted ORFs while the scaffolds representing the core O157:K88 734/3 genome consisted of 4,732 predicted ORFs. Both genomes displayed 94 RNAencoding ORFs. A BLASTn comparison, facilitated by Mauve, of the O157 SvETEC and O157:K88 734/3 genomes was performed on the scaffolds representing the core genomes of both ETEC O157 against the finished reference genome of E. coli APEC O78 (Figure 1). Common features between the two ETEC O157 core genomes included an enterotoxin-encoding scaffold and the transposon $\operatorname{Tn} 7$, which were not present within the reference genome. Scaffolds carrying genes encoding enterotoxins LT and STb aligned to core genome sequences of both strains, likely due to an IS911 gene aligning each scaffold to the same IS element in the APEC O78 genome sequence. BLASTn analysis of the LT/STb enterotoxin encoding scaffolds indicated similarity with pUMNK88 Ent [GenBank:CP002732] with both scaffolds exhibiting $99 \%$ sequence identity over $77 \%$ of the query sequence for O157 SvETEC and 79\% for O157:K88 734/3. These data suggest that the enterotoxin genes reside on a plasmid. Variability between the core O157 genomes was mostly limited to phage associated regions scattered throughout each genome alignment. Six phage-associated regions, designated Phage Regions S1-S6, were identified within the O157 SvETEC core genome and seven, designated Phage Regions K1-K7 were identified in the O157:K88 $734 / 3$ core genome (Table 1 and Figure 2). Two of the six phage regions in the O157 SvETEC genome were identified to be intact prophages by PHAST whereas five of the seven O157:K88 734/3 phage regions were identified as intact prophages. The majority of these phage associated scaffolds were putatively composed of $>85 \%$ phage and hypothetical gene content. Phage Region 1 of O157:K88 734/3 was most similar to a lambda phage and encoded the increased serum survival gene bor. Two identical phage-associated regions were shared by both $\mathrm{O} 157$ genomes. Phage Region 6 of O157 SvETEC and Phage Region 7 of O157:K88 734/3 were identical over $19.3 \mathrm{~kb}$. Phage Region 4 of O157 SvETEC was 99\% identical to Phage Region 5 of O157:K88 734/3. Two smaller regions in O157 SvETEC and one in O157:K88 734/3 were manually identified as phage associated regions and are represented by unlabelled boxes in Figure 2. In addition, the scaffold encoding the K88 adhesin operon in the O157: K88 734/3 genome also encoded several phage-associated genes that were lacking in the homologous O157 SvETEC scaffold. As such, this scaffold was aligned into the O157: K88 734/3 core genome due to these phage-associated genes. Variation between all three genome sequences was also observed around the APEC O78 O-antigen biosynthesis genes.

The accessory genome scaffolds of O157 SvETEC and O157:K88 734/3 were examined for ORFs. O157 SvETEC comprises 1008 predicted ORFs over 112 scaffolds including 8 RNA predictions, whereas O157:K88 734/3's comprises 851 predicted ORFs over 97 scaffolds including 8 RNA predictions. To further assess variability between the two genomes a comparative BLASTp analysis of the predicted amino acid sequence of the accessory genome ORFs of O157 SvETEC and O157:K88 734/3 was performed (Additional file 1: Table S1). Several scaffolds and consequently their predicted peptide sequences were determined to be unique to $\mathrm{O} 157$ SvETEC. The majority of genes common between the accessory genomes were plasmid related and included tra operon genes associated with IncF and IncI plasmids, and hypothetical proteins. Fragments of complex antimicrobial resistance loci (CRL) were also shared between the two accessory genomes including a Tn21-like mercury 


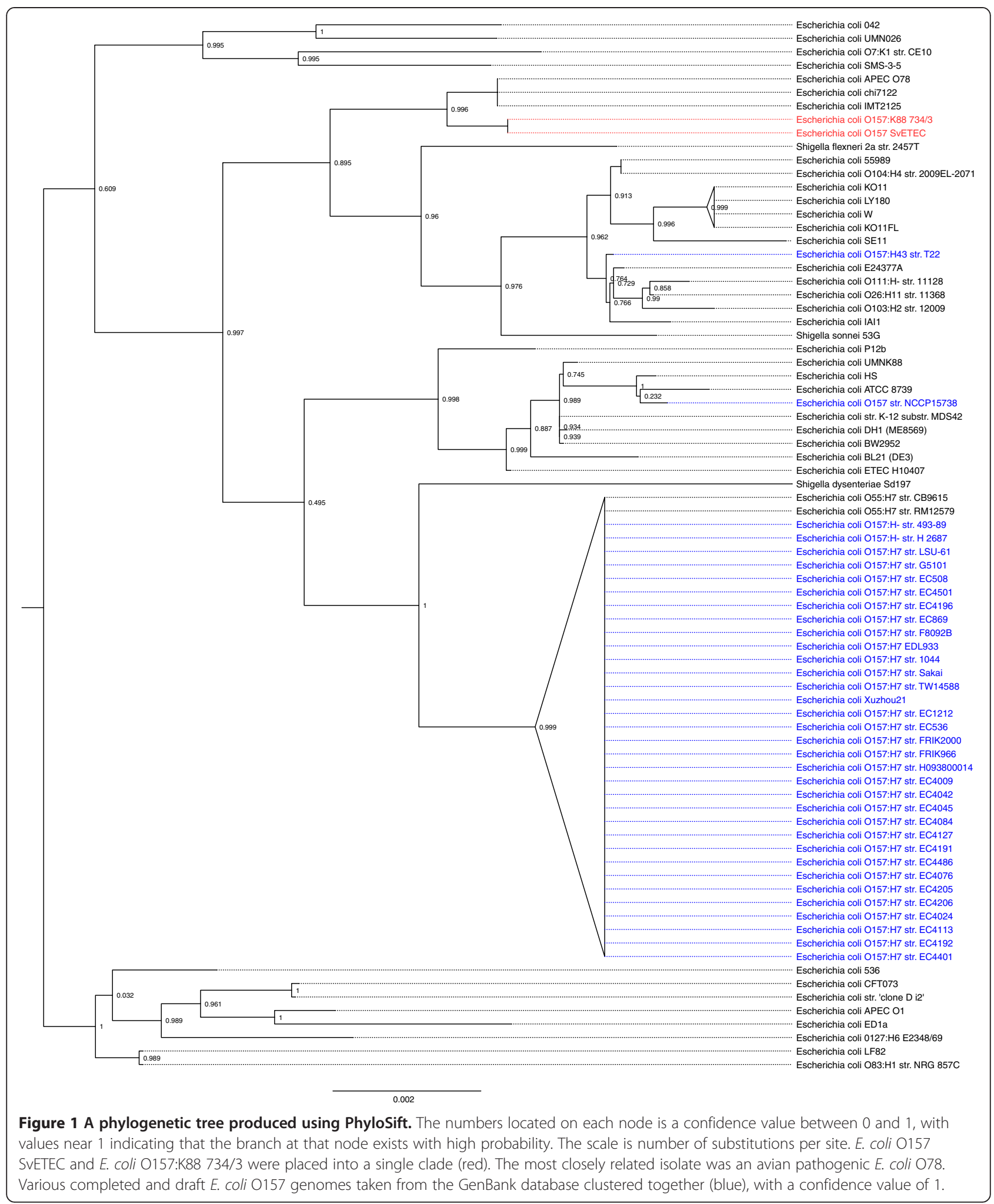

resistance operon and scaffolds associated with clinical class 1 and class 2 integrons. Several phage-containing or phage gene-associated scaffolds were also identified within the accessory genomes. Two approximately identical scaffolds of $29133 \mathrm{nt}$ and $29044 \mathrm{nt}$ respectively for O157 SvETEC and O157:K88 734/3 were identified as most similar to Enterobacteria phage P1 (NC_005856) but were predicted to be incomplete by PHAST. Both accessory 
Table 1 Phage associated regions identified by PHAST

\begin{tabular}{|c|c|c|c|c|c|c|c|}
\hline Strain & Region & $\begin{array}{l}\text { Concatenated }{ }^{1} \\
\text { Boundaries (bp) } \\
\end{array}$ & Size (kb) & $\begin{array}{l}\text { Ordered scaffold } \\
\text { numbers }\end{array}$ & PHAST Annotation & 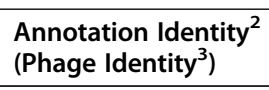 & $\begin{array}{l}\text { PHAST } \\
\text { Prediction }^{4} \\
\end{array}$ \\
\hline \multirow[t]{6}{*}{$\begin{array}{l}\text { E. coli O157 } \\
\text { SVETEC }\end{array}$} & S1 & $1316571-1334538$ & 17.9 & $83,114,2$ & $\begin{array}{l}\text { Stx2 converting I } \\
\text { NC_003525 }\end{array}$ & $24 \%(92 \%)$ & Incomplete \\
\hline & S2 & 2204749-2280987 & 76.2 & $59,58,107,53,39$ & mEp460 NC_019716 & $22 \%(90 \%)$ & Intact \\
\hline & S3 & 2966791-2982739 & 15.9 & $32,81,1$ & P1 NC_005856 & $50 \%(85.7 \%)$ & Incomplete \\
\hline & S4 & $3287635-3331585$ & 43.9 & 1,11 & TL 2011b NC_019445 & $78.57 \%$ (98.2\%) & Intact \\
\hline & S5 & $3449037-3477233$ & 28.1 & $44,70,14$ & P1 NC_005856 & $39.39 \%$ (84.8\%) & Incomplete \\
\hline & S6 & $4542510-4561835$ & 19.3 & 31 & APSE 2 NC_011551 & $15.78 \%(47.3 \%)$ & Incomplete \\
\hline \multirow{7}{*}{$\begin{array}{l}\text { E. coli O157:K88 } \\
734 / 3\end{array}$} & K1 & 1322304-1357135 & 34.8 & $67,55,141,6$ & lambda NC_001416 & $54.76 \%(97.6 \%)$ & Intact \\
\hline & K2 & 1555282-1584506 & 29.2 & $34,83,58$ & Fels 2 NC_010463 & $57.14 \%(100 \%)$ & Incomplete \\
\hline & K3 & 1810316-1849764 & 39.4 & 19,41 & mEp460 NC_019716 & $17.94 \%(92.3 \%)$ & Intact \\
\hline & K4 & $2254746-2314331$ & 59.5 & $\begin{array}{l}54,65,94,98 \\
132,136,38\end{array}$ & mEp460 NC_019716 & $29.11 \%(92.4 \%)$ & Intact \\
\hline & K5 & $3315282-3359241$ & 43.9 & 2,23 & TL 2011b NC_019445 & $78.18 \%$ (98.1\%) & Intact \\
\hline & K6 & $3476711-3497698$ & 20.9 & 30 & Fels 2 NC_010463 & $86.2 \%(100 \%)$ & Intact \\
\hline & K7 & $4558357-4577682$ & 19.3 & 7 & APSE 2 NC_011551 & $15 \%(45 \%)$ & Incomplete \\
\hline
\end{tabular}

${ }^{1}$ Identified regions were generally over multiple scaffolds but were identified as concatenated sequence. ${ }^{2}$ Percent identity of the region compared to the PHAST annotation. ${ }^{3}$ Percentage of identified ORFs that encode phage or hypothetical genes. ${ }^{4} \mathrm{PHAST}$ prediction of whether each prophage encodes the genes necessary for lysogeny.

genomes encoded plasmid transfer genes relating to the plasmid incompatibility groups F and I, however the O157 SvETEC accessory scaffolds also encoded IncH plasmidassociated genes. BLASTn analysis of the $66 \mathrm{~kb}$ (Scaffold 26) encoding IncH 1 plasmid-associated genes identified seven GenBank entries each with 96-99\% query coverage and $98-99 \%$ identity with the scaffold. Each entry was of a plasmid and included Serratia marcescens plasmid R478 [GenBank:BX664015], Salmonella enterica subsp. enterica Serovar Cubana str. CFSAN002050 plasmid [GenBank:CP006056] and Escherichia coli APEC O1 plasmid pAPEC-O1-R [GenBank:DQ517526]. Several other scaffolds within the O157 SvETEC accessory genome also aligned with high identity to these plasmids. These scaffold generally encoded hypothetical proteins with the exception of a copper/heavy metal (cus/czc) resistance operon (Scaffold 43) and a tellurium/tellurite (ter) resistance operon (Scaffold 35).

The O157 SvETEC accessory genome was also characterised by the presence of a Tn7-like transposon operon, hygromycin resistance, the enterotoxin $\mathrm{STa}$ and a second allele of STb, all of which were lacking in the O157: K88 734/3 accessory genome. A $17.8 \mathrm{~kb}$ region of another accessory O157:K88 734/3 scaffold was identified to contain an incomplete phage most similar to Enterobacteria phage P1 (NC_005856). Further BLASTn analysis

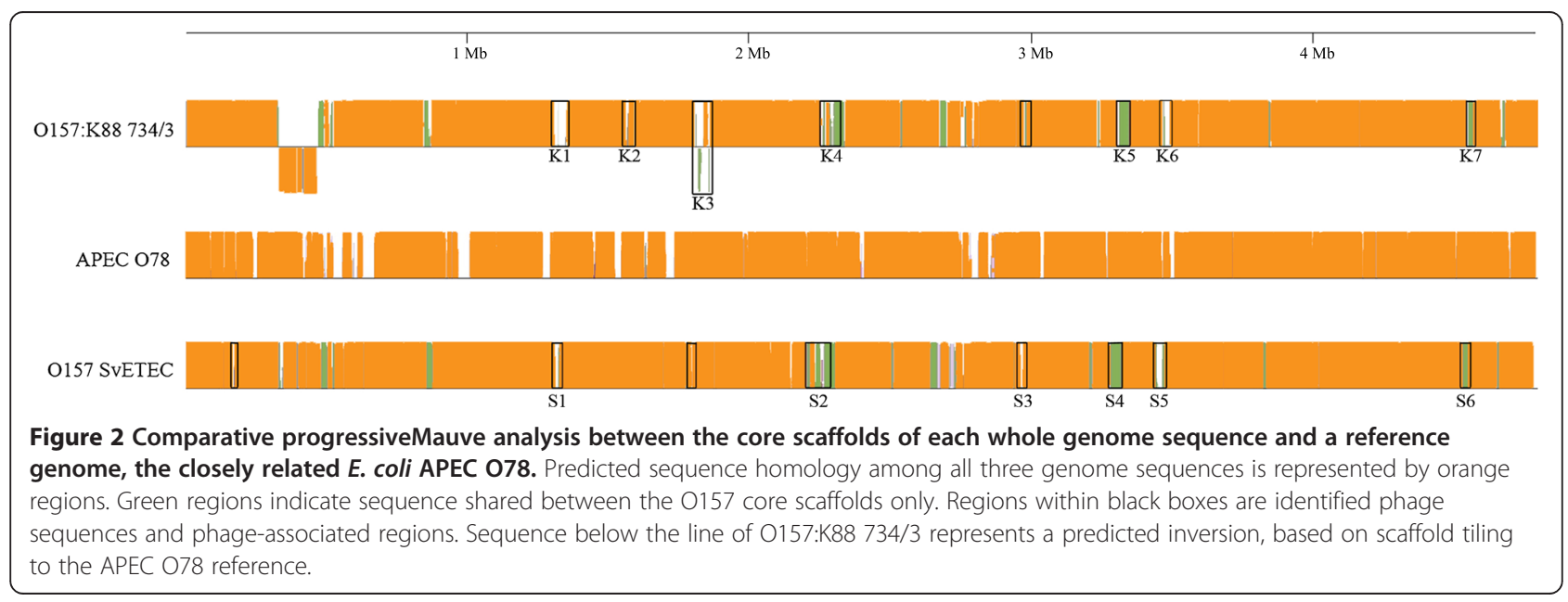


suggested both ETEC O157 genomes contained only a single copy of the P1-like phage, which has been split amongst various core and accessory scaffolds due to the reference tiling. Aside from these specifics, much of the variation between the accessory genomes of the two strains lies in plasmid associated hypothetical proteins, mobile elements and CRL-associated scaffolds.

The O-antigen biosynthesis genes within both ETEC O157 draft genome sequences were split across several scaffolds making detailed analysis difficult. Despite this, high synteny was observed between the O157-antigen encoding scaffolds with both isolates encoding the Sakai-type $r f b E$ gene (gene truncated at 1032 bp by a scaffold break in O157:K88 734/3) and also containing the genes yoeB and yefM identical to the E. coli strain PV00-24 O-antigen biosynthesis gene cluster [GenBank: AB602253.1][8].

\section{Virulence factors}

As both the ETEC O157 pathogens belong to the novel ST4245 sequence type we were particularly interested to identify potential virulence determinants within their genomes. Those we identified through sequence analysis are detailed in Table 2, including a variety of adhesin and toxin genes and their associated operons. Both O157 SvETEC and O157:K88 734/3 were positive for the enterotoxins LTp (eltAB) and STb (estB) whereas the heat stable enterotoxin STa (estA) was only identified in $\mathrm{O} 157$
SvETEC. A further distinction highlighted by sequence analysis was that a second allele of est $B$ was present in a separate scaffold within the O157 SvETEC genome. This allele differed by a single nucleotide polymorphism and was identical to the STb gene of Escherichia coli F18+ strain EC2173 plasmid pTC1 [GenBank:CP000913]. The $\mathrm{fliC}$ gene was, in both isolates, the $\mathrm{H} 19$ allele.

\section{Antimicrobial resistances}

Multiple antibiotic resistances were observed in the phenotypic analysis of O157 SvETEC and O157:K88 734/3. Both isolates were resistant to ampicillin, nalidixic acid, sulphafurazole, trimethoprim, tetracycline and neomycin. Each isolate displayed distinct additional resistance phenotypes against apramycin and chloramphenicol for O157 SvETEC and against cephalexin and cefoxitin for the O157:K88 $734 / 3$ isolate [4]. Various genes known to provide these antimicrobial resistances were identified within the genome sequences (Table 3). The gyrA sequence of both isolates was determined to contain a single S83L amino acid change compared with the $E$. coli K12 substrain MG1655 gyrA gene.

\section{Discussion}

In this study, we were able to characterise the evolutionary background of two non-EHEC E. coli O157 strains, and identify genetic differences between these two ETEC

Table 2 Virulence genes associated with pathogenicity present in ETEC 0157

\begin{tabular}{|c|c|c|c|c|}
\hline Type & Gene & O157 SvETEC & 0157:K88 734/3 & Details \\
\hline \multirow[t]{7}{*}{ Adhesins } & faeG & + & + & K88 (F4) Fimbrial adhesin \\
\hline & $\mathrm{fimH}$ & + & + & Type 1 fimbriae, D-Mannose-specific adhesin \\
\hline & $\operatorname{csg} G$ & + & + & Facilitator of fibronectin-binding curli assembly \\
\hline & eaeH & + & + & Highly conserved adhesin \\
\hline & $s f m A$ & + & + & Fimbrial-like adhesin \\
\hline & eсpA & + & + & E. coli common pilus \\
\hline & mat & + & + & mat operon \\
\hline \multirow[t]{8}{*}{ Toxins } & east1 & + & + & Enteroaggregative E. coli heat-stable enterotoxin \\
\hline & hlyA & + & + & a-Hemolysin \\
\hline & hlyE & + & + & Hemolysin E \\
\hline & eltB & + & + & Heat-labile enterotoxin B subunit \\
\hline & elt $A$ & + & + & Heat-labile enterotoxin A subunit \\
\hline & est $A$ & + & & Heat-stable enterotoxin a \\
\hline & estB & + & + & Heat-stable Enterotoxin b \\
\hline & estB & + & & Heat-stable Enterotoxin b (second allele) \\
\hline \multirow[t]{2}{*}{ Siderophores } & fyuA & + & + & Iron uptake receptor \\
\hline & $\operatorname{irp2}$ & + & + & Iron repressible protein \\
\hline \multirow[t]{3}{*}{ Other } & bor & & + & Serum survival \\
\hline & trat & + & + & Surface exclusion and serum survival \\
\hline & flic & + & + & Flagellin structural protein, $\mathrm{H}$ antigen determinant (H19 allele) \\
\hline
\end{tabular}


Table 3 Antibiotic and heavy metal resistance genes identified within ETEC 0157

\begin{tabular}{|c|c|c|c|c|}
\hline Resistance gene/Operon & O157 SvETEC & 0157:K88 734/3 & Phenotypic function/Resistance & Resistance observed \\
\hline$i n t / 1$ & + & + & Class 1 Integron Integrase & \\
\hline int $/ 2$ & + & + & Class 2 Integron Integrase & \\
\hline$b l a_{\mathrm{TEM}}$ & + & + & Ampicillin & + \\
\hline ampC & + & + & Cephalosporins & 0157:K88 734/3 only \\
\hline gyrA & + & + & Quinolones & + \\
\hline$a p h A 1$ & + & + & Aminoglycosides & + \\
\hline $\mathrm{APH}(4)-\mathrm{IA}$ & + & & Hygromycin B & O157 SvETEC only \\
\hline $\operatorname{str} A B$ & + & + & Streptomycin & + \\
\hline $\operatorname{aad} A$ & + & + & Streptomycin and Spectinomycin & + \\
\hline$d f r A$ & + & + & Trimethoprim & + \\
\hline aacc & + & & Apramycin & O157 SvETEC only \\
\hline sul1 & + & + & Sulphonamides & + \\
\hline sul2 & + & + & Sulphonamides & + \\
\hline sul3 & + & & Sulphonamides & + \\
\hline $\operatorname{mac} A B$ & + & + & Macrolides & \\
\hline tet operon & + & + & Tetracycline & + \\
\hline $\mathrm{cm} / \mathrm{A}$ & + & & Chloramphenicol & O157 SvETEC only \\
\hline emr operon & + & + & RND Efflux Pump & \\
\hline $\operatorname{gad} X$ & + & + & Acid Resistance Regulator & \\
\hline mdt operon & + & + & RND Efflux Pump & \\
\hline cus operon & + & + & Heavy Metals & \\
\hline mer operon & + & + & Mercury & \\
\hline ter operon & + & & Tellurium & \\
\hline
\end{tabular}

strains known to adopt different pathogenic strategies; $E$. coli O157:K88 734/3 associated with neonatal diarrhoea and E. coli O157 SvETEC associated with severe preand post-weaning disease. Our analysis demonstrated an evolutionary contrast between ETEC O157 and EHEC O157 and identified various small differences in toxin and antimicrobial resistance gene content between the two ETEC O157 isolates.

\section{Molecular evolution of ETEC 0157 strains}

The PhyloSift analysis demonstrated that the O157 SvETEC and O157:K88 734/3 ETEC isolates are most closely related to an APEC O78 isolate. This is interesting because previous work on APEC O78 isolates has reported such isolates to be closely related to human ETEC isolates [30]. PhlyoSift analysis also demonstrated a degree of relatedness between the ETEC O157 isolates, E. coli W and enterohaemorrhagic E. coli $\mathrm{O} 111: \mathrm{H}^{-}$. These results highlight the similarity of various $E$. coli pathotypes and serogroups when observed from a genomic perspective. In doing so these results further stress that clonal ancestry can play a minor role in predicting pathogenesis compared with the lateral acquisition of virulence factors. The PhyloSift analysis, which utilised the DNA sequences of 37 conserved marker genes, also demonstrated a contrast between the genetic relatedness of most E. coli O157: $\mathrm{H} 7$ genomes from the GenBank database and our ETEC O157:H19 genomes. Two O157 genomes proved exceptions to this observation; E. coli O157:H43 str. T22 from Hungary [31] and O157:H42 str. NCCP15738 from Korea (GenBank:ASHB01000001.1). Clustering of E. coli O157:H7 isolates and the divergence of non-H7 strains in the phylogenetic analysis supports the idea that $\mathrm{H}$-antigen typing may be a useful indicator of $E$. coli O157 lineage. In addition, the atypical (stx- and eae-negative) O157:H43 str. T22 encode CDT and long polar fimbriae [29], which underscores how O157 isolates carrying different $\mathrm{H}$-antigens may carry different repertoires of virulence genes.

Observations of $E$. coli relatedness similar to these have been made previously in relation to the parallel evolution of EHEC pathogenesis within the distantly related serogroups such as O157, O26 and O111 [32], where pathogenesis was mediated by similar laterally acquired elements such as plasmids and prophages. This phylogenetic divergence also suggests genetic recombination played a role in ETEC O157 development, with the ETEC O157 isolates encoding the "Sakai-type" O157 O-antigen biosynthesis 
gene cluster [8]. The acquisition of this biosynthesis gene cluster is an event known to have also shaped the evolution of enterohaemorrhagic E. coli O157 [33].

Multi-Locus Sequence Typing analysis of the O157 SvETEC and O157:K88 734/3 genomes supported their close evolutionary relationship to each other and to the ST23 complex which includes APEC O78. In the MLST database E. coli ST23 isolates have been sourced from avian, porcine, human and bovine hosts. Enterotoxigenic E. coli $\mathrm{O} 157$ strains of porcine origin from different geographic locations have been submitted to the MLST database as ST90 (ST23-complex) although we could find little mention or analysis of these strains in the literature.

\section{Comparison of ETEC 0157 core and accessory genomes}

Analysis of scaffolds representing the core genome from O157 SvETEC and O157:K88 734/3 highlighted the close relationship of these strains. Genetic differences were largely associated with the acquisition of prophageassociated sequences. Some prophages were common between the two isolates suggesting they may have been acquired prior to an evolutionary divergence. One phage in O157:K88 734/3 contained the bor gene which is known to be associated with increased serum survival. The bor gene has a lambda phage origin and is generally associated with avian extraintestinal pathogenic E. coli [34].

The variability of the accessory genomes for each isolate is mediated mostly by plasmid sequences, particularly IncH1 plasmid genes and heavy metal resistance operons, indicating that the differences in pathogenesis between these two isolates is essentially associated with plasmid content. Attempts to purify plasmids from our isolates using various plasmid extraction kits and standard alkaline lysis methods were unsuccessful. It is possible that the plasmids are extremely large, beyond the capability of the extraction methods used, or they are embedded in the chromosomes of these isolates. We were unable to confirm the presence of genomic islands in our analysis because of the reference genome based tiling exercise employed to separate the subset of scaffolds potentially encoding the core genome and accessory genomes. Large mass plasmids encoding resistance to multiple antibiotics in E. coli isolated from the faeces of food animals in Australia have been described [35,36] and as such may play a role in the pathogenesis of these isolates. Heavy metals are used frequently in pig production as antimicrobials and the identification of copper and mercury resistance loci in both strains and tellurium resistance in $\mathrm{O} 157$ SvETEC are likely provide significant survival advantages to these isolates. Antimicrobial resistance genes, discussed below, provide further advantages to these isolates. Such genes are often found on plasmids carrying mercury resistance transposons that serve as conduits for the lateral transfer of such genes $[37,38]$.

\section{Virulence factors}

E. coli virulence factors that are routinely screened for globally $[4,39,40]$ were identified in the O157 SvETEC and O157:K88 734/3 genomes. These included the porcine ETEC specific K88 fimbrial adhesin, and several adhesins commonly seen in other pathotypes of $E$. coli. The fim $H$ gene, which encodes a mannose-specific adhesin in extraintestinal pathogenic E. coli [41] has also been observed in commensal $E$. coli isolates or porcine origin [42]. BLAST analysis identified various adhesins, however most of these are common to E. coli isolates and have no definitive role in pathogenicity. The primary difference between the virulence profiles of the O157 SvETEC strain and the O157:K88 734/3 strain is that the latter lacks the heat-stable enterotoxin a (STa) and a second copy of STb. Links between the enterotoxins present in an isolate and the form of pathogenesis caused by ETEC have been described previously $[43,44]$ and these differences may impact pathogenesis. Previous work has also shown that the pTC plasmid-like variant of STb, which is homologous to the extra STb allele encoded in O157 SvETEC, is not enterotoxigenic in vivo $[45,46]$.

Other virulence associated genes identified in the O157 SvETEC and the O157:K88 734/3 genomes included the flagellin structural protein $f l i C$. The presence of the $f l i C$ gene, which encodes the H19 flagellar antigen [47], is important. Previous studies have demonstrated a correlation between the expression of flagellum and the K88ac fimbrial antigen [48] where the expression of both $f l i C$ and faeG, the major subunit of the K88 adhesin, correlates with adhesion to porcine epithelial cells in vitro. The characterisation of the flagellum also allows us to identify the serotype of both isolates as E. coli O157:H19:K88. Pathogenic $E$. coli of the serotype O157:H19 have been very sparingly reported [9] and studies involving isolates of this serotype have focussed on triclosan tolerance $[49,50]$. These studies do indicate that O157 strains have a capacity to adapt to multiple pathogenic lifestyles, having been identified as verocytotoxigenic, enterotoxigenic and enteropathogenic E. coli.

Another factor that appears to influence ETEC pathogenicity is the host's intestinal development and environment. Environmental conditions have an impact on enteroheamorrhagic E. coli pathogenesis and the regulation of virulence factor expression [51]. A similar situation has been observed in vitro for ETEC, with LT expression modified by $\mathrm{pH}$ [52]. Also, it has been demonstrated that the porcine intestinal epithelium can develop a resistance to F18-mediated adherence [53] and the stress of early weaning reduces the ability of piglets to cope with ETEC infection [54]. This data indicates that whilst it is important to characterise pathogenic $E$. coli, host characteristics and the intestinal environment may play a role in altering pathogenicity and account 
for the variation in disease severity between these isolates given their highly similar virulence factor content.

\section{Antibiotic resistance}

The greatest disparity between the O157 SvETEC and O157:K88 734/3 isolates is their antibiotic resistance. Both have quite comprehensive resistance profiles being resistant to beta-lactams, quinolones, aminoglycosides and cephalosporins. In context, one of the most important antimicrobial resistance genes in either strain is aphA1 which confers resistance to neomycin and kanamycin, with neomycin being a drug of choice for the treatment of infection during pig development within Australian pig farms. The majority of the observed resistance phenotypes have been accounted for including a known nalidixic acid resistance mutation in the DNA gyrase gene $g y r A$. Large complex antimicrobial gene resistance loci centred around both class 1 and class 2 integrons were identified within these genome sequences, however the prevalence of the insertion sequence IS26 in these regions precluded the automated assembly of the loci.

\section{Conclusion}

O157 SvETEC and O157:K88 734/3 appear to be part of a poorly characterised lineage of $E$. coli $\mathrm{O} 157$, which differs significantly from EHEC O157. Differences in pathogenicity between the strains may stem from differences in acquired virulence factors, antimicrobial resistance, and phage related genes (the majority of which are uncharacterised hypotheticals) with $E$. coli O157 SvETEC encoding a larger toxin repertoire compared to O157:K88 734/3. Further studies with these strains will focus on the assembly of antibiotic resistance gene loci and their association with mobile genetic elements.

\section{Additional file}

Additional file 1: A spreadsheet containing a BLASTp comparison of all identified open reading frames in the accessory genomes of both 0157 SvETEC and 0157:K88 734/3 draft genome sequences. Data includes scaffold numbers, ORF annotations and BLASTp homology as a percentage.

\section{Competing interests}

The authors declare that they have no competing interests.

\section{Authors' contributions}

SPD, TC, AD, SA, IC conceived the study. PRC and EW conducted the comparative genomic analyses. JS prepared the libraries for genome sequencing. SA and TC conducted initial PCR and phenotypic testing for antibiotic resistance. SPD, TC, EW, PRC, AD, SA and IC wrote the manuscript. All authors read and approved the final manuscript.

Authors' information

Piklu Roy Chowdhury is a co-first author.

\section{Acknowledgements}

This work was supported by collaboration between the NSW Department of Primary Industries and the ithree institute at the University of Technology Sydney. The authors would like to thank Fiona Maclver for her help in editing this manuscript.

\section{Author details}

'The ithree institute, University of Technology Sydney, P.O. Box 123, Broadway, NSW 2007, Australia. ${ }^{2}$ NSW Department of Primary Industries, Elizabeth Macarthur Agricultural Institute, Private Bag 4008, Narellan, NSW 2567, Australia. ${ }^{3}$ School of Animal and Veterinary Sciences, University of Adelaide, Adelaide, South Australia 5371, Australia.

Received: 3 September 2014 Accepted: 23 February 2015

Published online: 10 March 2015

\section{References}

1. Fairbrother JM, Nadeau E, Gyles CL. Escherichia coli in postweaning diarrhea in pigs: an update on bacterial types, pathogenesis, and prevention strategies. Anim Health Res Rev. 2005;6(1):17-39.

2. Smith MG, Jordan D, Chapman TA, Chin JJ, Barton MD, Do TN, et al. Antimicrobial resistance and virulence gene profiles in multi-drug resistant enterotoxigenic Escherichia coli isolated from pigs with post-weaning diarrhoea. Vet Microbiol. 2010;145(3-4):299-307.

3. Nagy B, Fekete PZ. Enterotoxigenic Escherichia coli (ETEC) in farm animals. Vet Res. 1999;30(2-3):259-84.

4. Abraham S, Trott DJ, Jordan D, Gordon DM, Groves MD, Fairbrother JM, et al. Phylogenetic and molecular insights into the evolution of multidrug-resistant porcine enterotoxigenic Escherichia coli in Australia. Int J Antimicrobial Agents. 2014;44(2):105-11.

5. Hayashi T, Makino K, Ohnishi M, Kurokawa K, Ishii K, Yokoyama K, et al. Complete genome sequence of enterohemorrhagic Escherichia coli 0157: $\mathrm{H} 7$ and genomic comparison with a laboratory strain K-12. DNA Res. 2001;8(1):11-22.

6. Pierard D, De Greve H, Haesebrouck F, Mainil J. O157:H7 and 0104:H4 Vero/Shiga toxin-producing Escherichia coli outbreaks: respective role of cattle and humans. Vet Res. 2012:43(1):13.

7. Rahal EA, Kazzi N, Nassar FJ, Matar GM. Escherichia coli 0157:H7-Clinical aspects and novel treatment approaches. Front Cell Infect Microbiol. 2012;2:138.

8. Iguchi A, Shirai H, Seto K, Ooka T, Ogura Y, Hayashi T, et al. Wide distribution of O157-antigen biosynthesis gene clusters in Escherichia coli. PLoS One. 2011;6(8):e23250.

9. Blanco M, Lazo L, Blanco JE, Dahbi G, Mora A, Lopez C, et al. Serotypes, virulence genes, and PFGE patterns of enteropathogenic Escherichia coli isolated from Cuban pigs with diarrhea. Int Microbiol. 2006:9(1):53-60.

10. Blank TE, Lacher DW, Scaletsky IC, Zhong H, Whittam TS, Donnenberg MS. Enteropathogenic Escherichia coli 0157 strains from Brazil. Emerg Infect Dis. 2003;9(1):113-5.

11. Toth I, Schmidt H, Kardos G, Lancz Z, Creuzburg K, Damjanova I, et al. Virulence genes and molecular typing of different groups of Escherichia coli 0157 strains in cattle. Appl Environ Microbiol. 2009;75(19):6282-91.

12. Frydendahl K. Prevalence of serogroups and virulence genes in Escherichia coli associated with postweaning diarrhoea and edema disease in pigs and a comparison of diagnostic approaches. Vet Microbiol. 2002;85(2):169-82.

13. Chapman TA, Wu XY, Barchia I, Bettelheim KA, Driesen S, Trott D, et al. Comparison of virulence gene profiles of Escherichia coli strains isolated from healthy and diarrheic swine. Appl Environ Microbiol. 2006;72(7):4782-95.

14. Do T, Stephens C, Townsend K, Wu X, Chapman T, Chin J, et al. Rapid identification of virulence genes in enterotoxigenic Escherichia coli isolates associated with diarrhoea in Queensland piggeries. Aust Vet J. 2005;83(5):293-9

15. Zhang W, Zhao M, Ruesch L, Omot A, Francis D. Prevalence of virulence genes in Escherichia coli strains recently isolated from young pigs with diarrhea in the US. Vet Microbiol. 2007:123(1-3):145-52.

16. Oh KH, Kim DW, Jung SM, Cho SH. Molecular characterization of enterotoxigenic Escherichia coli strains isolated from diarrheal patients in Korea during 2003-2011. PLoS One. 2014;9(5):e96896.

17. Guerra JA, Romero-Herazo YC, Arzuza O, Gomez-Duarte OG. Phenotypic and genotypic characterization of enterotoxigenic Escherichia coli clinical 
isolates from northern Colombia, South America. BioMed Res Int. 2014;2014:236260

18. Maynard C, Fairbrother JM, Bekal S, Sanschagrin F, Levesque RC, Brousseau $R$, et al. Antimicrobial resistance genes in enterotoxigenic Escherichia coll 0149:K91 isolates obtained over a 23-year period from pigs. Antimicrob Agents Chemother. 2003;47(10):3214-21

19. Abraham S, Chin J, Brouwers HJ, Zhang R, Chapman TA. Molecular serogrouping of porcine enterotoxigenic Escherichia coli from Australia. J Microbiol Methods. 2012;88(1):73-6.

20. Tritt A, Eisen JA, Facciotti MT, Darling AE. An integrated pipeline for de novo assembly of microbial genomes. PLoS One. 2012;7(9):e42304.

21. Wu XY, Chapman T, Trott DJ, Bettelheim K, Do TN, Driesen S, et al. Comparative analysis of virulence genes, genetic diversity, and phylogeny of commensal and enterotoxigenic Escherichia coli isolates from weaned pigs. Appl Environ Microbiol. 2007;73(1):83-91.

22. Aziz RK, Bartels D, Best AA, DeJongh M, Disz T, Edwards RA, et al. The RAST Server: rapid annotations using subsystems technology. BMC Genomics. 2008;9:75.

23. McArthur AG, Waglechner N, Nizam F, Yan A, Azad MA, Baylay AJ, et al. The comprehensive antibiotic resistance database. Antimicrob Agents Chemother. 2013;57(7):3348-57.

24. Zhou Y, Liang Y, Lynch KH, Dennis JJ, Wishart DS. PHAST: a fast phage search tool. Nucleic Acids Res. 2011;39(Web Server issue):W347-52.

25. Darling AE, Jospin G, Lowe E, Matsen FA, Bik HM, Eisen JA. PhyloSift: phylogenetic analysis of genomes and metagenomes. PeerJ. 2014;2:e243.

26. Price MN, Dehal PS, Arkin AP. FastTree 2-approximately maximum-likelihood trees for large alignments. PLoS One. 2010;5(3):e9490.

27. Darling AE, Mau B, Perna NT. ProgressiveMauve: multiple genome alignment with gene gain, loss and rearrangement. PLoS One. 2010;5(6):e11147.

28. Wirth T, Falush D, Lan R, Colles F, Mensa P, Wieler LH, et al. Sex and virulence in Escherichia coli: an evolutionary perspective. Mol Microbiol. 2006;60(5):1136-51.

29. Clermont O, Christenson JK, Denamur E, Gordon DM. The Clermont Escherichia coli phylo-typing method revisited: improvement of specificity and detection of new phylo-groups. Environ Microbiol Rep. 2013;5(1):58-65.

30. Dziva F, Hauser H, Connor TR, van Diemen PM, Prescott G, Langridge GC, et al. Sequencing and functional annotation of avian pathogenic Escherichia coli serogroup 078 strains reveal the evolution of E. coli lineages pathogenic for poultry via distinct mechanisms. Infect Immun. 2013;81(3):838-49.

31. Svab D, Horvath B, Szucs A, Maroti G, Toth I. Draft Genome sequence of an Escherichia coli 0157:H43 strain isolated from cattle. Genome Announc. 2013;1:3.

32. Ogura $Y$, Ooka T, Iguchi $A$, Toh $H$, Asadulghani M, Oshima $K$, et al. Comparative genomics reveal the mechanism of the parallel evolution of 0157 and non-0157 enterohemorrhagic Escherichia coli. Proc Natl Acad Sci U S A. 2009;106(42):17939-44.

33. Wick LM, Qi W, Lacher DW, Whittam TS. Evolution of genomic content in the stepwise emergence of Escherichia coli 0157:H7. J Bacteriol 2005;187(5):1783-91

34. Johnson TJ, Wannemuehler YM, Nolan LK. Evolution of the iss gene in Escherichia coli. Appl Environ Microbiol. 2008;74(8):2360-9.

35. Venturini C, Beatson SA, Djordjevic SP, Walker MJ. Multiple antibiotic resistance gene recruitment onto the enterohemorrhagic Escherichia coli virulence plasmid. FASEB J. 2010:24(4):1160-6.

36. Venturini C, Hassan KA, Roy Chowdhury P, Paulsen IT, Walker MJ, Djordjevic SP. Sequences of two related multiple antibiotic resistance virulence plasmids sharing a unique IS26-related molecular signature isolated from different Escherichia coli pathotypes from different hosts. PLoS One. 2013;8(11):e78862.

37. Roy Chowdhury P, McKinnon J, Wyrsch E, Hammond JM, Charles IG, Djordjevic SP: Genomic interplay in bacterial communities: implications for growth promoting practices in animal husbandry. Frontiers in Microbiol 2014, 5 .

38. Djordjevic SP, Stokes HW, Roy Chowdhury P. Mobile elements, zoonotic pathogens and commensal bacteria: conduits for the delivery of resistance genes into humans, production animals and soil microbiota. Front Microbiol. 2013:4:86

39. Comery R, Thanabalasuriar A, Garneau P, Portt A, Boerlin P, Reid-Smith RJ, et al. Identification of potentially diarrheagenic atypical enteropathogenic
Escherichia coli strains present in Canadian food animals at slaughter and in retail meats. Appl Environ Microbiol. 2013;79(12):3892-6.

40. DebRoy C, Maddox CW. Identification of virulence attributes of gastrointestinal Escherichia coli isolates of veterinary significance. Anim Health Res Rev. 2001;2(2):129-40.

41. Yun KW, Kim HY, Park HK, Kim W, Lim IS. Virulence factors of uropathogenic Escherichia coli of urinary tract infections and asymptomatic bacteriuria in children. J Microbiol Immunol Infect. 2014;47(6):455-61.

42. Bok E, Mazurek J, Pusz P, Stosik M, Baldy-Chudzik K. Age as a factor influencing diversity of commensal E. coli microflora in pigs. Pol J Microbiol. 2013;62(2):165-71.

43. Nakazawa M, Sugimoto C, Isayama Y, Kashiwazaki M. Virulence factors in Escherichia coli isolated from piglets with neonatal and post-weaning diarrhea in Japan. Vet Microbiol. 1987;13(4):291-300.

44. Erume J, Berberov EM, Kachman SD, Scott MA, Zhou Y, Francis DH, et al. Comparison of the contributions of heat-labile enterotoxin and heat-stable enterotoxin b to the virulence of enterotoxigenic Escherichia coli in F4ac receptor-positive young pigs. Infect Immun. 2008;76(7):3141-9.

45. Olasz F, Fekete PZ, Blum-Oehler G, Boldogkoi Z, Nagy B. Characterization of an F18+ enterotoxigenic Escherichia coli strain from post weaning diarrhoea of swine, and of its conjugative virulence plasmid pTC. FEMS Microbiol Lett. 2005:244(2):281-9

46. Fekete PZ, Brzuszkiewicz E, Blum-Oehler G, Olasz F, Szabo M, Gottschalk G, et al. DNA sequence analysis of the composite plasmid pTC conferring virulence and antimicrobial resistance for porcine enterotoxigenic Escherichia coli. Int J Med Microbiol. 2012;302(1):4-9.

47. Wang $L$, Rothemund D, Curd $H$, Reeves PR. Species-wide variation in the Escherichia coli flagellin (H-antigen) gene. J Bacteriol. 2003;185(9):2936-43.

48. Zhou M, Duan Q, Zhu X, Guo Z, Li Y, Hardwidge PR, et al. Both flagella and F4 fimbriae from F4ac + enterotoxigenic Escherichia coli contribute to attachment to IPEC-J2 cells in vitro. Vet Res. 2013:44(1):30.

49. Sheridan A, Lenahan M, Condell O, Bonilla-Santiago R, Sergeant K, Renaut J, et al. Proteomic and phenotypic analysis of triclosan tolerant verocytotoxigenic Escherichia coli 0157:H19. J Proteomics. 2013;80C:78-90.

50. Lenahan M, Sheridan A, Morris D, Duffy G, Fanning S, Burgess CM. Transcriptomic analysis of triclosan-susceptible and -tolerant Escherichia coli 0157:H19 in response to triclosan exposure. Microb Drug Resist. 2014;20(2):91-103.

51. Barnett Foster D. Modulation of the enterohemorrhagic E. coli virulence program through the human gastrointestinal tract. Virulence. 2013;4(4):315-23.

52. Gonzales L, Ali ZB, Nygren E, Wang Z, Karlsson S, Zhu B, et al. Alkaline pH Is a signal for optimal production and secretion of the heat labile toxin, LT in enterotoxigenic Escherichia coli (ETEC). PLoS One. 2013;8(9):e74069.

53. Zhu J, Zi C, Wu ZC, Liu L, Zheng XR, Su XM, et al. Age-dependent expression of the BPI gene in Sutai piglets. Genet Mol Res. 2013;12(2):2120-6.

54. McLamb BL, Gibson AJ, Overman EL, Stahl C, Moeser AJ. Early weaning stress in pigs impairs innate mucosal immune responses to enterotoxigenic E. coli challenge and exacerbates intestinal injury and clinical disease. PLoS One. 2013;8(4):e59838

\section{Submit your next manuscript to BioMed Central and take full advantage of:}

- Convenient online submission

- Thorough peer review

- No space constraints or color figure charges

- Immediate publication on acceptance

- Inclusion in PubMed, CAS, Scopus and Google Scholar

- Research which is freely available for redistribution 